\title{
Counting the number of elements in the mutation classes of $\tilde{A}_{n}$-quivers
}

\author{
Janine Bastian \\ Institut für Algebra, Zahlentheorie und Diskrete Mathematik \\ Leibniz Universität Hannover \\ Welfengarten 1, D-30167 Hannover, Germany \\ bastian@math. uni-hannover.de \\ Thomas Prellberg \\ School of Mathematical Sciences \\ Queen Mary University of London \\ Mile End Road, London E1 4NS, United Kingdom \\ t.prellberg@qmul.ac.uk \\ Martin Rubey \\ Institut für Algebra, Zahlentheorie und Diskrete Mathematik \\ Leibniz Universität Hannover \\ Welfengarten 1, D-30167 Hannover, Germany \\ martin.rubey@math . uni-hannover.de

\section{Christian Stump} \\ Laboratoire de combinatoire et d'informatique mathématique \\ Université du Québec à Montréal \\ Président-Kennedy, Montréal (Québec) H2X 3Y7, Canada \\ christian.stump@lacim.ca
}

Submitted: Oct 17, 2010; Accepted: Apr 20, 2011; Published: Apr 29, 2011

Mathematics Subject Classification: 05A15 16G20

\begin{abstract}
In this article we prove explicit formulae for the number of non-isomorphic cluster-tilted algebras of type $\tilde{A}_{n}$ in the derived equivalence classes. In particular, we obtain the number of elements in the mutation classes of quivers of type $\tilde{A}_{n}$. As a by-product, this provides an alternative proof for the number of quivers mutation equivalent to a quiver of Dynkin type $D_{n}$ which was first determined by Buan and Torkildsen in [5].
\end{abstract}




\section{Introduction}

Quiver mutation is a central element in the recent theory of cluster algebras introduced by Fomin and Zelevinsky in [10]. It is an elementary operation on quivers which generates an equivalence relation. The mutation class of a quiver $Q$ is the class of all quivers which are mutation equivalent to $Q$.

The mutation class of quivers of type $A_{n}$ is the class containing all quivers mutation equivalent to a quiver whose underlying graph is the Dynkin diagram of type $A_{n}$, shown in Figure 1(a). This mutation class was described by Caldero, Chapoton and Schiffler [7] in terms of triangulations. An explicit characterisation of the quivers themselves can be found in Buan and Vatne in [6]. The corresponding task for type $D_{n}$, shown in Figure 1(b), was accomplished by Vatne in [12]. Furthermore, an explicit formula for the number of quivers in the mutation class of type $A_{n}$ was given by Torkildsen in [11] and of type $D_{n}$ by Buan and Torkildsen in [5].

In this article, we consider quivers of type $\tilde{A}_{n-1}$. That is, all quivers mutation equivalent to a quiver whose underlying graph is the extended Dynkin diagram of type $\tilde{A}_{n-1}$, i.e., the $n$-cycle, see Figure $1(c)$. If this cycle is oriented, then we get the mutation class of $D_{n}$, see Fomin et al. in [9] and Type IV in [12]. If the cycle is non-oriented, we get the mutation classes of $\tilde{A}_{n-1}$, studied by the first named author in [2]. The purpose of this paper is to give an explicit formula for the number of quivers in the mutation classes of quivers of type $\tilde{A}_{n-1}$.

A cluster-tilted algebra $C$ of type $\tilde{A}_{n-1}$ is finite dimensional over an algebraically closed field $K$. Therefore, there exists a quiver $Q$ which is in one of the mutation classes of $\tilde{A}_{n-1}$ (see for instance Buan, Marsh and Reiten [4] or Assem et al. [1]) and an admissible ideal I of the path algebra $K Q$ of $Q$ such that $C \cong K Q / I$. Furthermore, two cluster-tilted algebras of the same type are isomorphic if and only if the corresponding quivers are isomorphic as directed graphs.

Thus, we also obtain the number of non-isomorphic cluster-tilted algebras of type $\tilde{A}_{n-1}$. In fact, we prove a more refined counting theorem. Namely, one can classify these algebras up to derived equivalence, see [2]. Each equivalence class is determined by four parameters, $r_{1}, r_{2}, s_{1}$ and $s_{2}$, where $r_{1}+2 r_{2}+s_{1}+2 s_{2}=n$, up to interchanging $r_{1}, r_{2}$ and $s_{1}, s_{2}$. Without loss of generality, we can therefore assume that $r_{1}<s_{1}$ or $r_{1}=s_{1}$ and $r_{2} \leq s_{2}$. Given positive integers $r$ and $s$ with $r+s=n$, the set of equivalence classes with $r_{1}+2 r_{2}=r$ and $s_{1}+2 s_{2}=s$ corresponds to one mutation class of quivers.

Theorem. The number of cluster-tilted algebras in the derived equivalence classes 
with parameters $r_{1}, r_{2}, s_{1}$ and $s_{2}$ is given by

$$
\begin{aligned}
& \sum_{k|r, k| r_{2}, k|s, k| s_{2}} \frac{\phi(k)}{k}(-1)^{\left(r+r_{2}+s+s_{2}\right) / k} \\
& \sum_{\substack{i, j \geq 0 \\
(i, j) \neq(0,0)}}\left[\frac{(-1)^{i+j}}{2(i+j)}\left(\begin{array}{c}
2 i \\
i, 2 i-r / k, r_{2} / k,\left(r-r_{2}\right) / k-i
\end{array}\right)\right. \\
&\left.\quad\left(\begin{array}{c}
2 j \\
j, 2 j-s / k, s_{2} / k,\left(s-s_{2}\right) / k-j
\end{array}\right)\right]
\end{aligned}
$$

if $r_{1}<s_{1}$ or $r_{1}=s_{1}$ and $r_{2}<s_{2}$. Otherwise, if $r_{1}=s_{1}$ and $r_{2}=s_{2}$, the number is

$$
\begin{aligned}
& 2^{r-2 r_{2}-2}\left(\begin{array}{c}
r \\
r_{2}, r_{2}, r-2 r_{2}
\end{array}\right) \\
&+\sum_{\substack{\left.k|r, k| r_{2} \\
i, j \geq 0 \\
i, j\right) \neq(0,0)}}\left[\frac{\phi(k)}{k} \frac{(-1)^{i+j}}{4(i+j)}\left(\begin{array}{c}
2 i \\
i, 2 i-r / k, r_{2} / k,\left(r-r_{2}\right) / k-i
\end{array}\right)\right. \\
&\left.\quad\left(\begin{array}{c}
2 j \\
j, 2 j-r / k, r_{2} / k,\left(r-r_{2}\right) / k-j
\end{array}\right)\right] .
\end{aligned}
$$

Here $\phi(k)$ is Euler's totient function, i.e., the number of $1 \leq d<k$ coprime to $k$ and $\left(\begin{array}{c}m \\ m_{1}, m_{2}, \ldots, m_{\ell}\end{array}\right)$ with $m_{1}+m_{2}+\cdots+m_{\ell}=m$ denotes the multinomial coefficient.

In particular, for $r=r_{1}+2 r_{2}$ and $s=s_{1}+2 s_{2}$, we obtain the number $\tilde{a}(r, s)$ of quivers mutation equivalent to a non-oriented $n$-cycle with $r$ arrows oriented in one direction and $s$ arrows oriented in the other direction:

$$
\tilde{a}(r, s)= \begin{cases}\frac{1}{2} \sum_{k|r, k| s} \frac{\phi(k)}{r+s}\left(\begin{array}{c}
2 r / k \\
r / k
\end{array}\right)\left(\begin{array}{c}
2 s / k \\
s / k
\end{array}\right) & \text { if } r<s, \\
\frac{1}{2}\left(\frac{1}{2}\left(\begin{array}{c}
2 r \\
r
\end{array}\right)+\sum_{k \mid r} \frac{\phi(k)}{4 r}\left(\begin{array}{c}
2 r / k \\
r / k
\end{array}\right)^{2}\right) & \text { if } r=s .\end{cases}
$$

Additionally, we obtain the number of quivers in the mutation class of a quiver of Dynkin type $D_{n}$. This formula was first determined in [5]:

Corollary. The number of quivers of type $D_{n}$, for $n \geq 5$, is given by

$$
\tilde{a}(0, n)=\sum_{d \mid n} \frac{\phi(n / d)}{2 n}\left(\begin{array}{c}
2 d \\
d
\end{array}\right) .
$$

The number of quivers of type $D_{4}$ is 6 . 

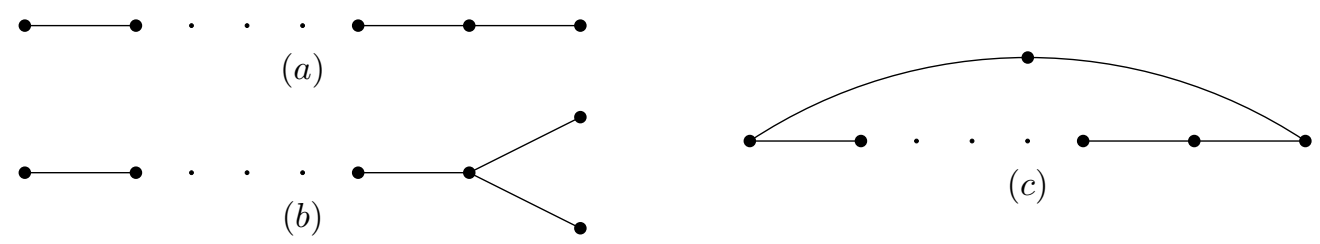

(c)

Figure 1: The Dynkin diagrams of types $A_{n}$ and $D_{n}$ and the extended Dynkin diagram of type $\tilde{A}_{n-1}$, assuming that all diagrams have $n$ vertices.

The paper is organized as follows. In Section 2 we collect some basic notions about quiver mutation. Furthermore, we present the classification of quivers of type $\tilde{A}_{n-1}$ according to the parameters $r$ and $s$ mentioned above, as given in [2]. In Section 3 we restate the classification as a combinatorial grammar. Using 'generatingfunctionology' we obtain the formulae for the assymmetric case where $r_{1} \neq s_{1}$ or $r_{2} \neq s_{2}$. For the case $r_{1}=s_{1}$ and $r_{2}=s_{2}$ some additional combinatorial considerations, counting the number of quivers invariant under reflection, yield the result stated above.

The formulae for $\tilde{a}(r, s)$ are developed in parallel. In fact, it is remarkable that the generating function including variables for the parameters $r_{2}$ and $s_{2}$ can be obtained by specialising the much simpler generating function having variables for the parameters $r$ and $s$ only. Moreover, extracting the coefficient of $p^{r} q^{s} x^{r_{2}} y^{s_{2}}$ in a naive way from the equations obtained from combinatorial grammars results in a much uglier five-fold sum, instead of the three-fold sum stated in the main theorem.

Finally, at the end of Section 3 we prove the formula for the number of quivers in the mutation class of type $D_{n}$, by exhibiting an appropriate bijection between these and a subclass of the objects counted in Section 3.2.

Acknowledgements: We would like to thank Thorsten Holm for invaluable comments on a preliminary version of this article, and Ira Gessel for providing the beautiful proof of Lemma 3.3. We also would like to thank Christian Krattenthaler who gave an elementary proof of the same lemma, of which at first we only had a computer assisted proof.

\section{Preliminaries}

A quiver $Q$ is a (finite) directed graph where loops and multiple arrows are allowed. Formally, $Q$ is a quadruple $Q=\left(Q_{0}, Q_{1}, h, t\right)$ consisting of two finite sets $Q_{0}, Q_{1}$ 
whose elements are called vertices and arrows resp., and two functions

$$
h: Q_{1} \rightarrow Q_{0}, \quad t: Q_{1} \rightarrow Q_{0}
$$

assigning a head $h(\alpha)$ and a tail $t(\alpha)$ to each arrow $\alpha \in Q_{1}$.

Moreover, if $t(\alpha)=i$ and $h(\alpha)=j$ for $i, j \in Q_{0}$, we say $\alpha$ is an arrow from $i$ to $j$ and write $i \stackrel{\alpha}{\rightarrow} j$. In this case, $i$ and $\alpha$ as well as $j$ and $\alpha$ are called incident to each other. As usual, two quivers are considered to be equal if they are isomorphic as directed graphs. The underlying graph of a quiver $Q$ is the graph obtained from $Q$ by replacing the arrows in $Q$ by undirected edges.

A quiver $Q^{\prime}=\left(Q_{0}^{\prime}, Q_{1}^{\prime}, h^{\prime}, t^{\prime}\right)$ is a subquiver of a quiver $Q=\left(Q_{0}, Q_{1}, h, t\right)$ if $Q_{0}^{\prime} \subseteq Q_{0}$ and $Q_{1}^{\prime} \subseteq Q_{1}$ and where $h^{\prime}(\alpha)=h(\alpha) \in Q_{0}^{\prime}, t^{\prime}(\alpha)=t(\alpha) \in Q_{0}^{\prime}$ for any arrow $\alpha \in Q_{1}^{\prime}$. A subquiver is called a full subquiver if for any two vertices $i$ and $j$ in the subquiver, the subquiver also will contain all arrows between $i$ and $j$ present in $Q$.

An oriented cycle is a subquiver of a quiver whose underlying graph is a cycle on at least two vertices and whose arrows are all oriented in the same direction, i.e., every vertex has outdegree 1. By contrast, a non-oriented cycle is a subquiver of a quiver whose underlying graph is a cycle, but not all of its arrows are oriented in the same direction.

Throughout the paper, unless explicitly stated, we assume that

- quivers do not have loops or oriented 2-cycles, i.e., $h(\alpha) \neq t(\alpha)$ for any arrow $\alpha$ and there do not exist arrows $\alpha, \beta$ such that $h(\alpha)=t(\beta)$ and $h(\beta)=t(\alpha)$;

- quivers are connected.

\subsection{Quiver mutation}

In [10], Fomin and Zelevinsky introduced the quiver mutation of a quiver $Q$ without loops and oriented 2-cycles at a given vertex of $Q$ :

Definition 2.1. Let $Q$ be a quiver. The mutation of $Q$ at a vertex $k$ is defined to be the quiver $Q^{*}:=\mu_{k}(Q)$ given as follows.

1. Add a new vertex $k^{*}$.

2. Suppose that the number of arrows $i \rightarrow k$ in $Q$ equals $a$, the number of arrows $k \rightarrow j$ equals $b$ and the number of arrows $j \rightarrow i$ equals $c \in \mathbb{Z}$. Then we have $c-a b$ arrows $j \rightarrow i$ in $Q^{*}$. Here, a negative number of arrows means arrows in the opposite direction. 
3. For any arrow $i \rightarrow k$ (resp. $k \rightarrow j$ ) in $Q$ add an arrow $k^{*} \rightarrow i\left(\right.$ resp. $\left.j \rightarrow k^{*}\right)$ in $Q^{*}$.

4. Remove the vertex $k$ and all its incident arrows.

No other arrows are affected by this operation.

Note that mutation at sinks or sources only means changing the direction of all incoming and outgoing arrows. Mutation at a vertex $k$ is an involution on quivers, that is, $\mu_{k}\left(\mu_{k}(Q)\right)=Q$. It follows that mutation generates an equivalence relation and we call two quivers mutation equivalent if they can be obtained from each other by a finite sequence of mutations. The mutation class of a quiver $Q$ is the class of all quivers (up to relabelling of the vertices) which are mutation equivalent to $Q$.

We have the following well-known lemma:

Lemma 2.2. If quivers $Q, Q^{\prime}$ have the same underlying graph which is a tree, then $Q$ and $Q^{\prime}$ are mutation equivalent.

This lemma implies that one can speak of quivers associated to a simply-laced Dynkin diagram, i.e., the Dynkin diagram of type $A_{n}, D_{n}$ or $E_{n}$ : we define a quiver of type $A_{n}$ (resp. $D_{n}, E_{n}$ ) to be a quiver in the mutation class of all quivers whose underlying graph is the Dynkin diagram of type $A_{n}$ (resp. $D_{n}, E_{n}$ ). We remark that some authors use this term to refer to an orientation of the Dynkin diagram of type $A_{n}$ (resp. $\left.D_{n}, E_{n}\right)$.

One can easily check that the oriented $n$-cycle is also of type $D_{n}$, as has been done in $[12$, Type IV]. Two non-oriented $n$-cycles are mutation equivalent if and only if the number of arrows oriented clockwise coincide, or the number of arrows oriented clockwise in one cycle agrees with the number of arrows oriented anti-clockwise in the other cycle. This was shown in $[2,9]$ and is restated in Theorem 2.10. We call quivers in those mutation classes quivers of type $\tilde{A}_{n-1}$. They will be described in more detail in Section 2.2. In Figure 1, the Dynkin diagrams of types $A_{n}$ and $D_{n}$ and the extended Dynkin diagram of $\tilde{A}_{n-1}$ are shown.

Example 2.3. The mutation class of type $\tilde{A}_{3}$ of the non-oriented cycles with two arrows in each direction is given by

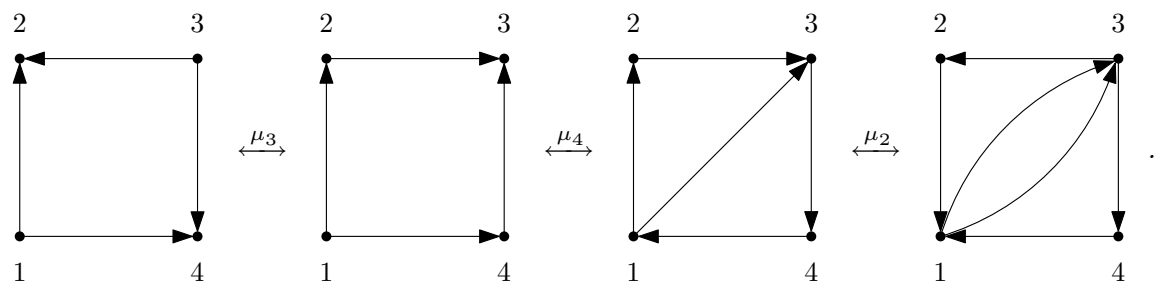


The mutation class of type $\tilde{A}_{3}$ of the non-oriented cycle with 3 arrows in one direction and 1 arrow in the other is given by

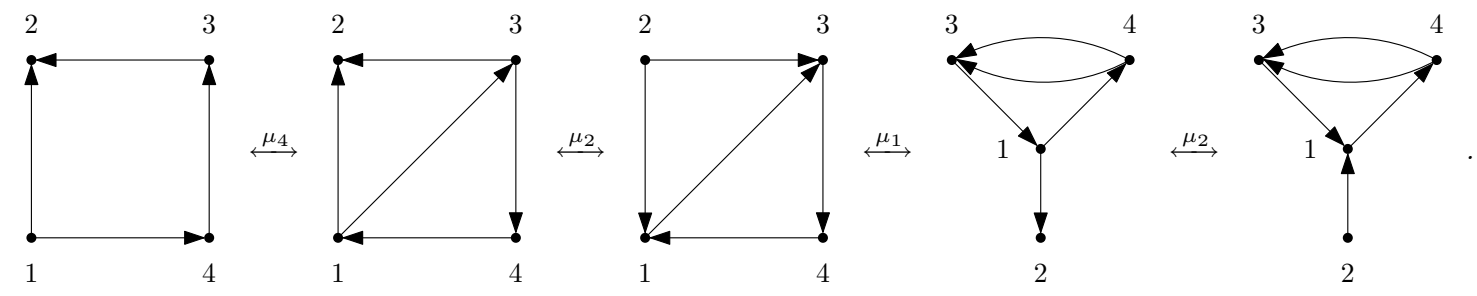

\subsection{Mutation classes of $\tilde{A}_{n-1}$-quivers}

Following [2], we now describe the mutation classes of quivers of type $\tilde{A}_{n-1}$ in more detail:

Definition 2.4. Let $\mathcal{Q}_{n-1}$ be the class of quivers with $n$ vertices which satisfy the following conditions:

1. There exists precisely one full subquiver which is a non-oriented cycle of length $\geq 2$. Thus, if the length is two, it is a double arrow.

2. For each arrow $x \stackrel{\alpha}{\rightarrow} y$ in this non-oriented cycle, there may (or may not) be a vertex $z_{\alpha}$ which is not on the non-oriented cycle, such that there is an oriented 3 -cycle of the form

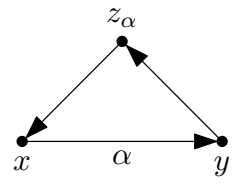

Apart from the arrows of these oriented 3-cycles there are no other arrows incident to vertices on the non-oriented cycle.

3. If we remove all vertices in the non-oriented cycle and their incident arrows, the result is a disconnected union of quivers, one for each $z_{\alpha}$. These are quivers of type $A_{k_{\alpha}}$ for $k_{\alpha} \geq 1$ (see [6] for the mutation class of $A_{n}$ ), and the vertices $z_{\alpha}$ have at most two incident arrows in these quivers. Furthermore, if a vertex $z_{\alpha}$ has two incident arrows in such a quiver, then $z_{\alpha}$ is a vertex in an oriented 3 -cycle. We call these quivers rooted quivers of type $A$ with root $z_{\alpha}$. Note that this is a similar description as for Type IV in [12].

The rooted quiver of type $A$ with root $z_{\alpha}$ is called attached to the arrow $\alpha$. 
Remark 2.5. Our convention is to choose only one of the double arrows to be part of the oriented 3-cycle in the following case:

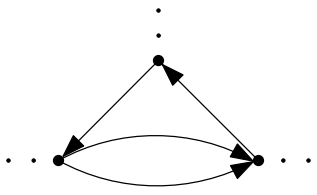

Example 2.6. The following quiver is of type $\tilde{A}_{21}$ :

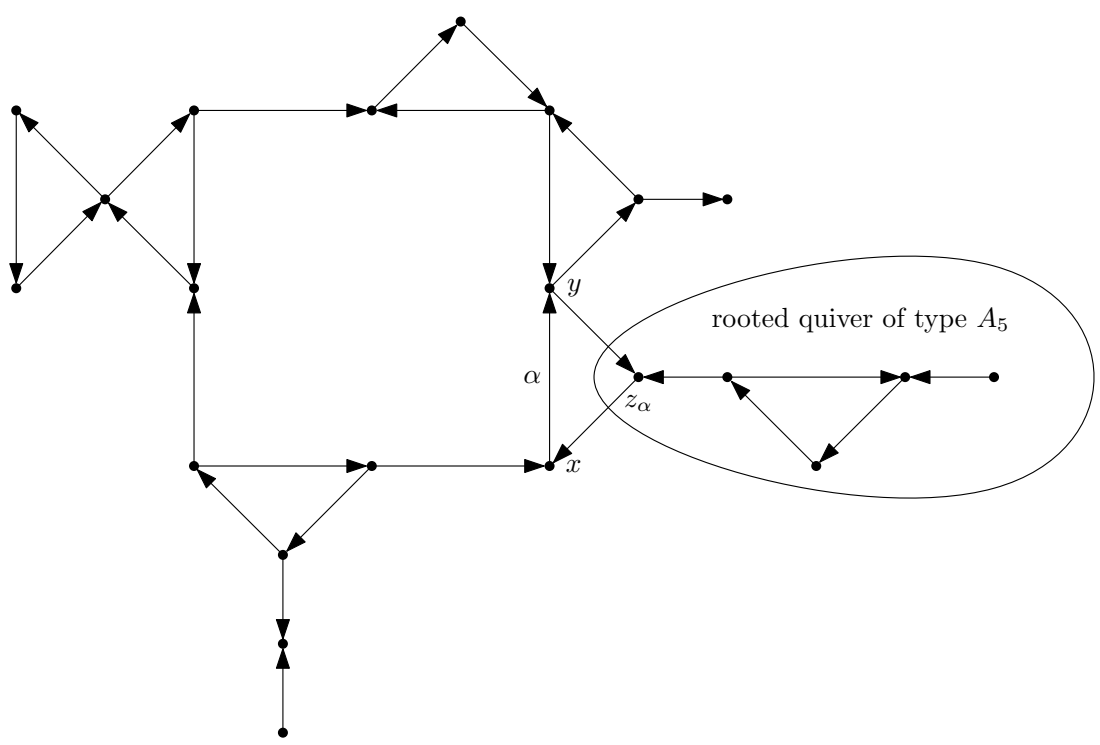

Definition 2.7. A realization of a quiver $Q \in \mathcal{Q}_{n-1}$ is the quiver together with an embedding of the non-oriented cycle into the plane. We do not care about a particular embedding of the other arrows, i.e., there are at most two different realizations of any given quiver. Thus, we can speak of clockwise and anti-clockwise oriented arrows in the non-oriented cycle.

We will see in Section 3 that it is straightforward to count the number of possible realizations of quivers in a mutation class of $\tilde{A}_{n-1}$. Since the two realizations of a quiver may coincide, we will need an additional argument to count the number of quivers themselves.

As in [2] we can define parameters $r_{1}, r_{2}, s_{1}$ and $s_{2}$ for a realization of a quiver $Q \in \mathcal{Q}_{n-1}$ as follows:

Definition 2.8. Let $Q$ be a quiver in $\mathcal{Q}_{n-1}$ and fix a realization of $Q$. The arrows in $Q$ which are part of the non-oriented cycle are called base arrows. Let $r_{1}$ be the number of arrows which are not part of any oriented 3-cycle and which are either 
1. base arrows and oriented anti-clockwise, or

2. contained in a rooted quiver of type $A$ attached to a base arrow $\alpha$ which is oriented anti-clockwise.
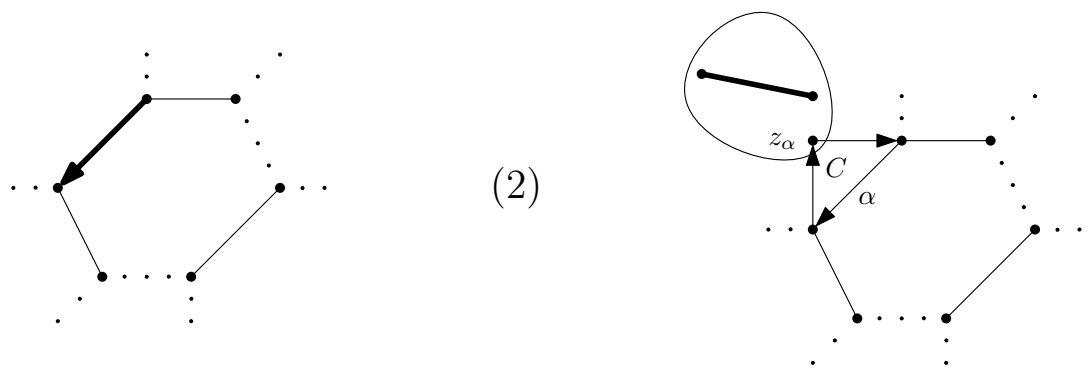

Let $r_{2}$ be the number of oriented 3-cycles

1. which share an arrow $\alpha$ with the non-oriented cycle and $\alpha$ (a base arrow) is oriented anti-clockwise, or

2. which are contained in a rooted quiver of type $A$ attached to a base arrow $\alpha$ which is oriented anti-clockwise.
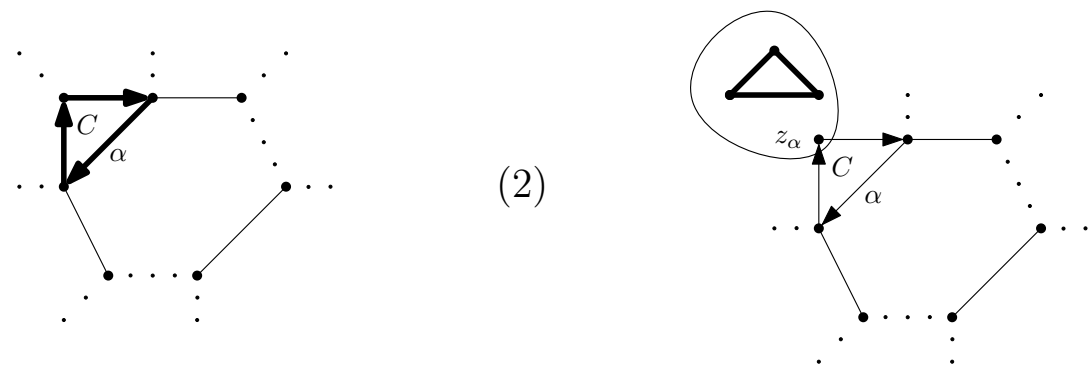

Similarly we define the parameters $s_{1}$ and $s_{2}$ with 'anti-clockwise' replaced by 'clockwise'.

Example 2.9. We indicate the arrows which count for the parameter $r_{1}$ by $\cdots . . . .$. and the arrows which count for $s_{1}$ by $\longrightarrow$. Furthermore, the oriented 3-cycles counting for $r_{2}$ are indicated by $\because$ : ${ }^{\circ}$ and the oriented 3 -cycles counting for $s_{2}$ are indicated by $\boldsymbol{\Delta}$.

Consider the following realization of a quiver in $\mathcal{Q}_{16}$ : 


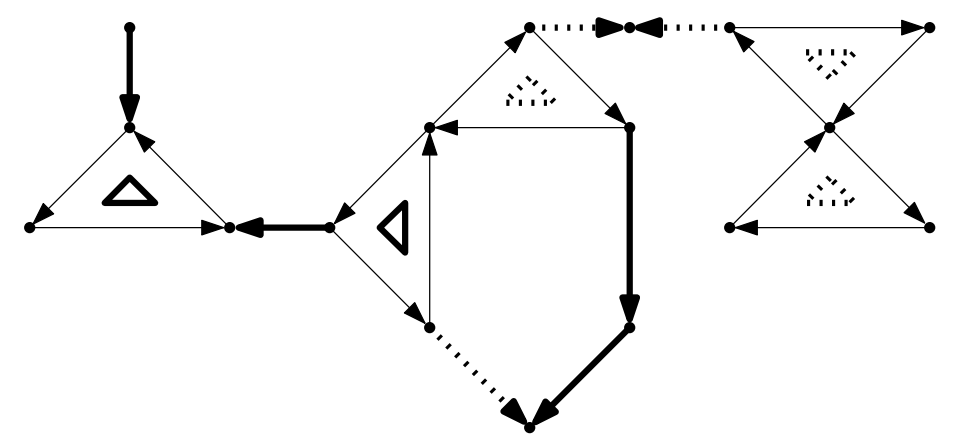

Here, we have $r_{1}=3, r_{2}=3, s_{1}=4$ and $s_{2}=2$.

In [2] an explicit description of the mutation classes of quivers of type $\tilde{A}_{n-1}$ and, moreover, the derived equivalence classes of cluster-tilted algebras of type $\tilde{A}_{n-1}$ is given as follows:

Theorem 2.10. [2, Theorem 3.12, Theorem 5.5] Let $Q_{1} \in \mathcal{Q}_{n-1}$ be a quiver with a realization having parameters $r_{1}, r_{2}, s_{1}$ and $s_{2}$ such that $r_{1}<s_{1}$ or $r_{1}=s_{1}$ and $r_{2} \leq s_{2}$. Similarly, let $Q_{2} \in \mathcal{Q}_{n-1}$ be a quiver with a realization having parameters $\tilde{r}_{1}, \tilde{r}_{2}, \tilde{s}_{1}$ and $\tilde{s}_{2}$ such that $\tilde{r}_{1}<\tilde{s}_{1}$ or $\tilde{r}_{1}=\tilde{s}_{1}$ and $\tilde{r}_{2} \leq \tilde{s}_{2}$. Then $Q_{1}$ is mutation equivalent to $Q_{2}$ if and only if $r_{1}+2 r_{2}=\tilde{r}_{1}+2 \tilde{r}_{2}$ and $s_{1}+2 s_{2}=\tilde{s}_{1}+2 \tilde{s}_{2}$.

Moreover, two cluster-tilted algebras of type $\tilde{A}_{n-1}$ are derived equivalent if and only if their quivers have realizations with the same parameters $r_{1}, r_{2}, s_{1}$ and $s_{2}$.

\section{A Combinatorial Grammar}

In this section we describe the elements of the mutation classes of type $\tilde{A}_{n-1}$ by a combinatorial grammar. This can be viewed as an exercise in the theory of species (introduced by Joyal, see the book [3] by Bergeron, Labelle and Leroux) or the symbolic method (as detailed in the recent book [8] by Flajolet and Sedgewick). We first give a recursive description of rooted quivers of type $A$ as defined in 2.4. A quiver of type $\tilde{A}_{n-1}$ will then be roughly a cycle of rooted quivers of type $A$.

\subsection{A recursive description of rooted quivers of type $A$}

Let $A^{\bullet}$ be the set of all rooted quivers of type $A$. We can then describe the elements of $A^{\bullet}$ recursively. A rooted quiver of type $A$ is one of the following:

- the root; 
- the root, incident to an arrow, and a rooted quiver of type $A$ incident to the other end of the arrow. The arrow may be directed either way.

- the root, incident to an oriented 3-cycle, and two rooted quivers of type $A$, each being incident to one of the other two vertices of the 3-cycle.

We obtain the following combinatorial grammar:
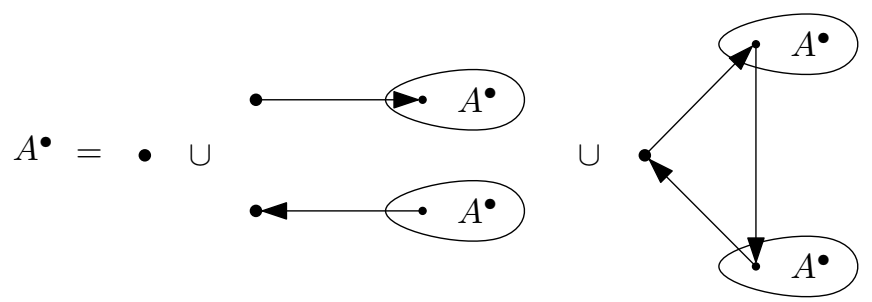

We set the weight of an arrow which is not part of an oriented 3-cycle equal to $z$ and the weight of an oriented 3-cycle equal to $t z^{2}$. Hence, the weight of a rooted quiver $Q$ of type $A$ is $z^{\#\{\text { vertices in } Q\}-1} t^{\# \text { \{riented 3-cycles in } Q\}}$. This choice of weight is in accordance with the first part of Theorem 2.10 where we count oriented 3-cycles in quivers (the number of which we denoted $r_{2}$, resp. $s_{2}$ ) twice.

Thus, let

$$
A^{\bullet}(z, t)=\sum_{Q \in A} z^{\#\{\text { vertices in } Q\}-1} t^{\#\{\text { oriented 3-cycles in } Q\}}
$$

be the generating function (in particular: the formal power series) associated to rooted quivers of type $A$. From the recursive description, we obtain

$$
A^{\bullet}(z, t)=1+2 z A^{\bullet}(z, t)+z^{2} t A^{\bullet}(z, t)^{2}
$$

or equivalently

$$
z^{2} t A^{\bullet}(z, t)^{2}+(2 z-1) A^{\bullet}(z, t)+1=0
$$

Solving this quadratic equation for $A^{\bullet}(z, t)$ and choosing the branch corresponding to a generating function gives

$$
A^{\bullet}(z, t)=\frac{1-2 z-\sqrt{1-4\left(z+(t-1) z^{2}\right)}}{2 z^{2} t} .
$$


We remark that for $t=1$ this is the generating function for the Catalan numbers shifted by 1 ,

$$
\begin{aligned}
A^{\bullet}(z, 1) & =\frac{1-2 z-\sqrt{1-4 z}}{2 z^{2}} \\
& =\sum_{n \geq 1} \frac{1}{n+1}\left(\begin{array}{c}
2 n \\
n
\end{array}\right) z^{n-1}
\end{aligned}
$$

see e.g. [3, Section 3.0 Eq. (3)].

To give a combinatorial description of the realizations of quivers in the mutation classes of type $\tilde{A}_{n-1}$ corresponding to Definition 2.8 we need auxiliary objects, which are one of the following:

1. a single (base) arrow, oriented from left to right, or

2. a rooted quiver of type $A$ attached to an oriented 3-cycle, whose base arrow (see Definition 2.8) is oriented from left to right, or

3. a single (base) arrow, oriented from right to left, or

4. a rooted quiver of type $A$ attached to an oriented 3-cycle, whose base arrow is oriented from right to left.

Remark 3.1. The 'base arrows' in (1)-(4) above will become precisely the arrows of the non-oriented cycle, which justifies the usage of the name.

Thus, we again obtain a combinatorial grammar:

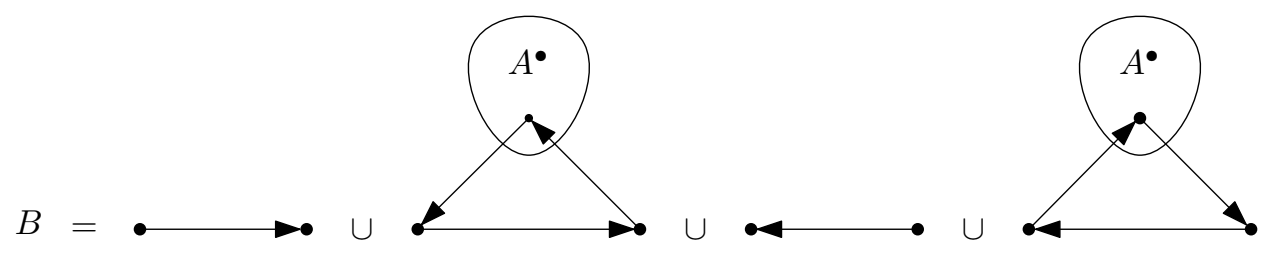

The weight of an object $Q \in B$ is $p^{\#\{\text { vertices in } Q\}-1} x^{\#\{\text { oriented 3-cycles in } Q\}}$ if it is of type (1) or (2), and $q^{\#\{\text { vertices in } Q\}-1} y^{\#\{\text { oriented 3-cycles in } Q\}}$ if it is of type (3) or (4). In particular, the weight of $Q$ depends only on the orientation of the base arrow and on the total number of vertices and 3-cycles of $Q$. Passing to generating functions, 
we obtain

$$
\begin{aligned}
B(p, q, x, y) & =p+p^{2} x A^{\bullet}(p, x)+q+q^{2} y A^{\bullet}(q, y) \\
& =\frac{1-\sqrt{1-4\left(p+(x-1) p^{2}\right)}}{2}+\frac{1-\sqrt{1-4\left(q+(y-1) q^{2}\right)}}{2} \\
& =\left(p+(x-1) p^{2}\right) C\left(p+(x-1) p^{2}\right)+\left(q+(y-1) q^{2}\right) C\left(q+(y-1) q^{2}\right),
\end{aligned}
$$

where $C(z)$ is the generating function for the Catalan numbers,

$$
C(z)=\frac{1-\sqrt{1-4 z}}{2 z}=\sum_{n \geq 0} \frac{1}{n+1}\left(\begin{array}{c}
2 n \\
n
\end{array}\right) z^{n} .
$$

Note that

$$
B(p, q, x, y)=B\left(p+(x-1) p^{2}, q+(y-1) q^{2}, 1,1\right)
$$

\subsection{The number of quivers of type $\tilde{A}_{n-1}$}

In this section we will first determine the number of realizations of quivers of type $\tilde{A}_{n-1}$, as defined in Definition 2.7. This already suffices to determine the number of quivers with parameters $r_{1}, r_{2}, s_{1}, s_{2}$ such that $r_{1}<s_{1}$ or $r_{1}=s_{1}$ and $r_{2}<s_{2}$, see Corollary 3.6. We then count quivers with $r_{1}=s_{1}$ and $r_{2}=s_{2}$ that are symmetric, i.e., whose two realizations coincide, to determine the number of quivers in the general case as stated in Corollary 3.9.

By Definition 2.4, a realization of a quiver of type $\tilde{A}_{n-1}$ is simply a cyclic arrangement of elements in $B$ with a total of $n$ vertices. For example, the quiver in Example 2.9 consists of five elements of $B$, three of which are just arrows, the two others are rooted quivers of type $A$ attached to an oriented 3-cycle.

The following Lemma is the so called cycle construction, which is well known in combinatorics, see eg. [3, Eq. (18), Section 1.4] or [8, Theorem I.1, Section I.2.2].

Lemma 3.2. Let $B(z)$ be the generating function for a family of unlabelled objects, where $z$ marks size. Then the generating function for cycles of such objects is

$$
\sum_{k \geq 1} \frac{\phi(k)}{k} \log \left(\frac{1}{1-B\left(z^{k}\right)}\right)
$$

where $\phi(k)$ is Euler's totient function, i.e., the number of $1 \leq d<k$ coprime to $k$.

The ELECTROnic Journal of COMbinatorics 15 (2008), \#R00 
Thus, we obtain for the generating function for realizations of quivers of type $\tilde{A}_{n-1}$ with $p$ marking $r_{1}+2 r_{2}, q$ marking $s_{1}+2 s_{2}, x$ marking $r_{2}$ and $y$ marking $s_{2}$

$$
\tilde{A}(p, q, x, y)=\sum_{k \geq 1} \frac{\phi(k)}{k} \log \left(\frac{1}{1-B\left(p^{k}, q^{k}, x^{k}, y^{k}\right)}\right) .
$$

Let us first determine the coefficients in the special case of $\log \frac{1}{1-B(p, q, 1,1)}$.

Lemma 3.3. For $(r, s) \neq(0,0)$ we have

$$
\left[p^{r} q^{s}\right] \log \left(\frac{1}{1-B(p, q, 1,1)}\right)=\frac{1}{2(r+s)}\left(\begin{array}{c}
2 r \\
r
\end{array}\right)\left(\begin{array}{c}
2 s \\
s
\end{array}\right),
$$

where $\left[p^{r} q^{s}\right] G(p, q)$ denotes the coefficient of $p^{r} q^{s}$ in the formal power series $G(p, q)$.

Proof. A direct calculation gives

$$
\begin{aligned}
1 & +2 t \frac{d}{d t} \log \left(\frac{1}{1-B(t p, t q, 1,1)}\right) \\
& =1+\frac{2 t}{\sqrt{1-4 t p}+\sqrt{1-4 t q}}\left(\frac{2 p}{\sqrt{1-4 t p}}+\frac{2 q}{\sqrt{1-4 t q}}\right) \\
& =\frac{1}{\sqrt{1-4 t p}+\sqrt{1-4 t q}}\left(\sqrt{1-4 t p}+\frac{4 t p}{\sqrt{1-4 t p}}+\sqrt{1-4 t q}+\frac{4 t q}{\sqrt{1-4 t q}}\right) \\
& =\frac{1}{\sqrt{1-4 t p}+\sqrt{1-4 t q}}\left(\frac{1}{\sqrt{1-4 t p}}+\frac{1}{\sqrt{1-4 t q}}\right) \\
& =\frac{1}{\sqrt{1-4 t p}} \cdot \frac{1}{\sqrt{1-4 t q}} \\
& =\sum_{r, s \geq 0}\left(\begin{array}{c}
2 r \\
r
\end{array}\right)\left(\begin{array}{c}
2 s \\
s
\end{array}\right) p^{r} q^{s} t^{r+s} .
\end{aligned}
$$

Denoting $a_{r, s}=\left[p^{r} q^{s}\right] \log \left(\frac{1}{1-B(p, q, 1,1)}\right)$ we have

$$
\begin{aligned}
1+2 t \frac{d}{d t} \log \left(\frac{1}{1-B(t p, t q, 1,1)}\right) & =1+2 t \frac{d}{d t} \sum_{r, s \geq 0} a_{r, s} p^{r} q^{s} t^{r+s} \\
& =1+2 \sum_{r, s \geq 0}(r+s) a_{r, s} p^{r} q^{s} t^{r+s} .
\end{aligned}
$$

We now obtain $a_{r, s}$ by equating coefficients on the right hand sides of Equation (1) and Equation (2). 
We can now determine the coefficients of $\log \frac{1}{1-B(p, q, x, y)}$.

\section{Lemma 3.4.}

$$
\begin{aligned}
& {\left[p^{r} q^{s} x^{r_{2}} y^{s_{2}}\right] \log \left(\frac{1}{1-B(p, q, x, y)}\right)} \\
& \begin{array}{r}
=(-1)^{r+r_{2}+s+s_{2}} \sum_{\substack{i, j \geq 0 \\
(i, j) \neq(0,0)}}\left[\frac{(-1)^{i+j}}{2(i+j)}\left(\begin{array}{c}
2 i \\
i, 2 i-r, r_{2}, r-r_{2}-i
\end{array}\right)\right. \\
\left.\left(\begin{array}{c}
2 j \\
j, 2 j-s, s_{2}, s-s_{2}-j
\end{array}\right)\right],
\end{array}
\end{aligned}
$$

where $\left[p^{r} q^{s} x^{r_{2}} y^{s_{2}}\right] B(p, q, x, y)$ denotes the coefficient of $p^{r} q^{s} x^{r_{2}} y^{s_{2}}$ in the formal power series $B(p, q, x, y)$.

Proof. From Lemma 3.3 and the substitution $B(p, q, x, y)=B\left(p+(x-1) p^{2}, q+(y-\right.$ 1) $\left.q^{2}, 1,1\right)$ it follows that

$\log \left(\frac{1}{1-B(p, q, x, y)}\right)=\sum_{\substack{i, j \geq 0 \\(i, j) \neq(0,0)}} \frac{1}{2(i+j)}\left(\begin{array}{c}2 i \\ i\end{array}\right)\left(\begin{array}{c}2 j \\ j\end{array}\right) p^{i}(1+(x-1) p)^{i} q^{j}(1+(y-1) q)^{j}$

A simple expansion gives now

$$
\begin{aligned}
\log & \left(\frac{1}{1-B(p, q, x, y)}\right) \\
= & \sum_{\substack{i, j \geq 0 \\
(i, j) \neq(0,0)}} \sum_{k, l, r_{2}, s_{2} \geq 0} p^{i+k} q^{j+l} x^{r_{2}} y^{s_{2}} \frac{(-1)^{k+r_{2}+l+s_{2}}}{2(i+j)}\left(\begin{array}{c}
2 i \\
i
\end{array}\right)\left(\begin{array}{c}
2 j \\
j
\end{array}\right)\left(\begin{array}{l}
i \\
k
\end{array}\right)\left(\begin{array}{l}
j \\
l
\end{array}\right)\left(\begin{array}{c}
k \\
r_{2}
\end{array}\right)\left(\begin{array}{c}
l \\
s_{2}
\end{array}\right) \\
= & \sum_{\substack{r, s, r_{2}, s_{2} \geq 0 \\
p^{r}}} q^{s} x^{r_{2}} y^{s_{2}} \\
& \sum_{\substack{i, j \geq 0 \\
(i, j) \neq(0,0)}} \frac{(-1)^{r+s+r_{2}+s_{2}+i+j}}{2(i+j)}\left(\begin{array}{c}
2 i \\
i
\end{array}\right)\left(\begin{array}{c}
2 j \\
j
\end{array}\right)\left(\begin{array}{c}
i \\
r-i
\end{array}\right)\left(\begin{array}{c}
j \\
s-j
\end{array}\right)\left(\begin{array}{c}
r-i \\
r_{2}
\end{array}\right)\left(\begin{array}{c}
s-j \\
s_{2}
\end{array}\right),
\end{aligned}
$$

from which one reads off the desired result.

Putting the pieces together we obtain: 
Theorem 3.5. The number of realizations of quivers of type $\tilde{A}_{r+s-1}$ with parameters $r>0$ and $s>0$ is given by

$$
\frac{1}{2} \sum_{k|r, k| s} \frac{\phi(k)}{r+s}\left(\begin{array}{c}
2 r / k \\
r / k
\end{array}\right)\left(\begin{array}{c}
2 s / k \\
s / k
\end{array}\right) .
$$

The number of realizations of quivers of type $\tilde{A}_{r+s-1}$ with parameters $r_{1}, r_{2}, s_{1}, s_{2}$ such that $r=r_{1}+2 r_{2}>0$ and $s=s_{1}+2 s_{2}>0$ is given by

$$
\begin{aligned}
& \sum_{k|r, k| r_{2}, k|s, k| s_{2}} \frac{\phi(k)}{k}(-1)^{\left(r+r_{2}+s+s_{2}\right) / k} \\
& \sum_{\substack{i, j \geq 0 \\
i, j) \neq(0,0)}} \frac{(-1)^{i+j}}{2(i+j)}\left(\begin{array}{c}
2 i \\
i, 2 i-r / k, r_{2} / k,\left(r-r_{2}\right) / k-i
\end{array}\right) \\
&\left.\quad\left(\begin{array}{c}
2 j \\
j, 2 j-s / k, s_{2} / k,\left(s-s_{2}\right) / k-j
\end{array}\right)\right] .
\end{aligned}
$$

Proof. Observe that for any $F(p, q)=\sum_{r, s} f_{r, s} p^{r} q^{s}$ we have

$$
\left[p^{r} q^{s}\right] F\left(p^{k}, q^{k}\right)= \begin{cases}f_{r / k, s / k} & \text { when } k \mid r \text { and } k \mid s \\ 0 & \text { otherwise }\end{cases}
$$

Using Lemma 3.3 we get

$$
\begin{aligned}
{\left[p^{r} q^{s}\right] \sum_{k \geq 1} \frac{\phi(k)}{k} \log \left(\frac{1}{1-B\left(p^{k}, q^{k}, 1,1\right)}\right) } & =\sum_{k \geq 1} \frac{\phi(k)}{k}\left[p^{r} q^{s}\right] \log \left(\frac{1}{1-B\left(p^{k}, q^{k}, 1,1\right)}\right) \\
& =\sum_{k|r, k| s} \frac{\phi(k)}{k} \frac{k}{2(r+s)}\left(\begin{array}{c}
2 r / k \\
r / k
\end{array}\right)\left(\begin{array}{c}
2 s / k \\
s / k
\end{array}\right) .
\end{aligned}
$$

The general formula follows similarly from Lemma 3.4.

As a corollary we obtain the number of quivers of type $\tilde{A}_{r+s-1}$ with parameters that do not coincide:

Corollary 3.6. For $r<s$, the number $\tilde{a}(r, s)$ of quivers of type $\tilde{A}_{r+s-1}$ with parameters $r$ and $s$ is given by Formula (3). For $r_{1}<s_{1}$ or $r_{1}=s_{1}$ and $r_{2}<s_{2}$, the number of quivers with parameters $r_{1}, r_{2}, s_{1}$ and $s_{2}$ is given by Formula (4).

Proof. If $r_{1}<s_{1}$ or $r_{1}=s_{1}$ and $r_{2}<s_{2}$, a quiver has a unique realization with these parameters. Therefore, the claim follows directly from Theorem 3.5. 
We have seen that a quiver of type $\tilde{A}_{2 r-1}$ is a non-oriented cycle of elements in $B$ with a total number of $2 r$ vertices. To count quivers of type $\tilde{A}_{2 r-1}$, we first have to consider symmetric quivers of type $\tilde{A}_{2 r-1}$, i.e., quivers where both possible realizations coincide. To do so, we have to count lists of elements in $B$ :

Lemma 3.7. The number of lists $\left(B_{1}, \ldots, B_{\ell}\right)$ of elements in $B$ with a total of $r+\ell$ vertices is given by the central binomial coefficient $\left(\begin{array}{c}2 r \\ r\end{array}\right)$. The number of such lists with $r_{2}$ oriented 3 -cycles is given by

$$
2^{r-2 r_{2}}\left(\begin{array}{c}
r \\
r_{2}, r_{2}, r-2 r_{2}
\end{array}\right)=2^{r-2 r_{2}}\left(\begin{array}{c}
r \\
2 r_{2}
\end{array}\right)\left(\begin{array}{c}
2 r_{2} \\
r_{2}
\end{array}\right) .
$$

Proof. The generating function for elements in $B$ taking into account only the number of vertices is $B(p, p, 1,1)=1-\sqrt{1-4 p}$. Thus, we obtain that the number of lists of elements in $B$ with $r+\ell$ vertices in total is given by

$$
\left[p^{r}\right] \frac{1}{1-B(p, p, 1,1)}=\left[p^{r}\right] \frac{1}{\sqrt{1-4 p}}=\left[p^{r}\right] \sum_{n \geq 0}\left(\begin{array}{c}
2 n \\
n
\end{array}\right) p^{n}=\left(\begin{array}{c}
2 r \\
r
\end{array}\right)
$$

compare [3, Example 1.2.2(a) and Theorem 1.4.2].

Let us now prove the more refined statement, by giving a meaning to each of the factors in the last expression of Equation (5). We first observe that $r_{1}=r-2 r_{2}$ is precisely the number of arrows that are not part of an oriented 3-cycle, and thus $2^{r-2 r_{2}}$ is the number of their possible orientations.

The central binomial coefficient $\left(\begin{array}{c}2 r_{2} \\ r_{2}\end{array}\right)$ can be interpreted as the number of lists $L^{B_{\Delta}}=\left(B_{1}, \ldots, B_{\ell}\right)$ of elements in $B$, where all elements consist of oriented 3-cycles only: namely, such a list is either empty, or its first element is an oriented 3-cycle (with its two possible orientations), to which a rooted quiver of type $A$, consisting of oriented 3 cycles only, is attached. It is easy to see that the generating function for such rooted quivers is $\frac{1-\sqrt{1-4 x}}{2 x}$. Let us denote the generating function for the lists under consideration $L^{B_{\Delta}}(x)$. We then have:

$$
\begin{aligned}
L^{B_{\Delta}}(x) & =1+2 x \frac{1-\sqrt{1-4 x}}{2 x} L^{B_{\Delta}}(x) \\
& =1+(1-\sqrt{1-4 x}) L^{B_{\Delta}}(x) \\
& =\frac{1}{\sqrt{1-4 x}} .
\end{aligned}
$$

Finally, $\left(\begin{array}{c}r \\ 2 r_{2}\end{array}\right)=\left(\begin{array}{c}\left(2 r_{2}+1\right)+r_{1}-1 \\ r_{1}\end{array}\right)$ is the number of ways to choose $r_{1}$ vertices (with repetitions) in a list $L^{B_{\Delta}}$ where arrows can be inserted to obtain a list of elements 


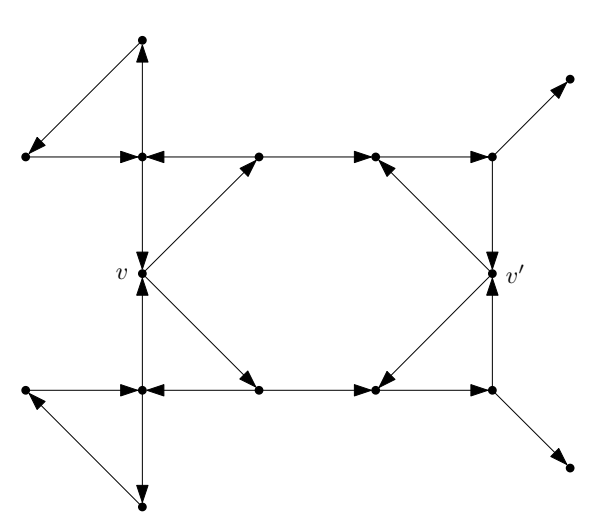

$(a)$

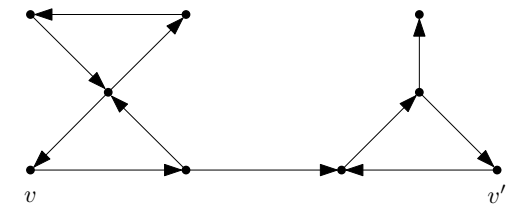

(b)

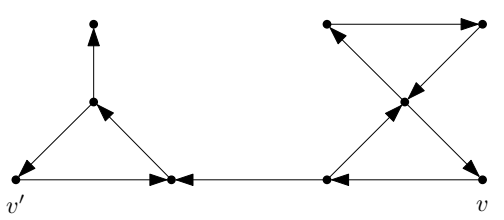

(c)

Figure 2: $(a)$ a symmetric quiver of type $\tilde{A}_{15} ;(b)$ the list $L$ of elements in $B$ starting at $v$ end ending at $v^{\prime} ;(c)$ the list $\operatorname{rev}(L)$ of elements in $B$ starting at $v^{\prime}$ end ending at $v$.

in $B$ with $r+\ell$ vertices and $r_{2}$ oriented 3 -cycles. Namely, there are $2 r_{2}+\ell$ vertices in total in $L^{B_{\Delta}}$, all but the $\ell-1$ vertices which are at the left of the base-arrows in $B_{2}, \ldots, B_{\ell}$ are possible insertion places.

Given a list $L=\left(B_{1}, \ldots, B_{\ell}\right)$ of elements in $B$, we identify $L$ with the quiver obtained from $L$ by gluing together the right vertex in the base arrow of $B_{i}$ and the left vertex in the base arrow of $B_{i+1}$ for $1 \leq i<\ell$. For a list $L=\left(B_{1}, \ldots, B_{\ell}\right)$ of elements in $B$ define the reversed list $\operatorname{rev}(L):=\left(\bar{B}_{\ell}, \ldots, \bar{B}_{1}\right)$, where $\bar{B}_{i}$ is obtained from $B_{i}$ by reversing the direction of the base arrow of $B_{i}$ (and eventually of the associated oriented 3-cycle). See Figures $2(b)$ and $2(c)$ for an example. Obviously, we have $\operatorname{rev}(\operatorname{rev}(L))=L$.

Theorem 3.8. The number of symmetric quivers of type $\tilde{A}_{2 r-1}$, i.e., quivers where both possible realizations coincide, is equal to $\frac{1}{2}\left(\begin{array}{c}2 r \\ r\end{array}\right)$. The number of symmetric quivers of type $\tilde{A}_{2 r-1}$ with $2 r_{2}$ oriented 3 -cycles is

$$
2^{r-2 r_{2}-1}\left(\begin{array}{c}
r \\
r_{2}, r_{2}, r-2 r_{2}
\end{array}\right)
$$

Proof. Starting with a list $L$ of elements in $B$ with a total of $r+\ell$ vertices, we obtain a symmetric quiver of type $\tilde{A}_{2 r-1}$ by taking $L$ and $\operatorname{rev}(L)$, and gluing together the end point of $L$ with the start point of $\operatorname{rev}(L)$ and vice versa. E.g., the symmetric quiver in Figure 2(a) is obtained from the lists $L$ and $\operatorname{rev}(L)$ shown in Figures 2(b) and $2(c)$. 
To prove the statement it remains to show that exactly two different lists belong to the given symmetric quiver $Q$. Observe first, that $Q$ is of the form $Q=\left(L, L^{\prime}\right)$ where the end point of $L$ is glued together with the start point of $L^{\prime}$ and vice versa, such that furthermore, $L^{\prime}=\operatorname{rev}(L)$ is the reversed list of $L$. It may happen that $L$ is itself symmetric, i.e., $L=\operatorname{rev}(L)$. However, it is always possible to find a non-symmetric $X$ such that $Y:=\operatorname{rev}(X) \neq X$ and $L=(X, Y, X, \ldots, Y)$ and $L^{\prime}=(\bar{Y}, \bar{X}, \bar{Y}, \ldots, \bar{X})$. That is, any symmetric quiver is of the following form:

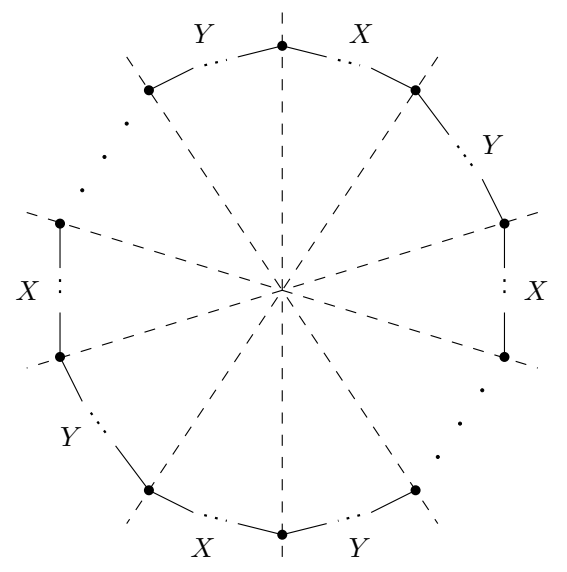

This proves that there exist exactly two different lists that correspond to a symmetric quiver $Q$, namely $L$ and $L^{\prime}$.

We now know the number of realizations of quivers as well as the number of symmetric quivers of type $\tilde{A}_{2 r-1}$ with parameters $r$ and $s=r$. Therefore, we can also compute the total number of quivers of type $\tilde{A}_{2 r-1}$ with the same parameters:

Corollary 3.9. The number $\tilde{a}(r, r)$ of quivers of type $\tilde{A}_{2 r-1}$ with parameters $r$ and $s=r$ is given by

$$
\frac{1}{2}\left(\frac{1}{2}\left(\begin{array}{c}
2 r \\
r
\end{array}\right)+\sum_{k \mid r} \frac{\phi(k)}{4 r}\left(\begin{array}{c}
2 r / k \\
r / k
\end{array}\right)^{2}\right) .
$$

The number of quivers of type $\tilde{A}_{2 r-1}$ with parameters $r_{1}, r_{2}, s_{1}$ and $s_{2}$ such that 


\begin{tabular}{|c|c|c|c|c|c|}
\hline 2 & 1 & & & & \\
\hline 3 & 2 & & & & \\
\hline 4 & 5 & 4 & & & \\
\hline 5 & 14 & 12 & & & \\
\hline 6 & 42 & 36 & 22 & & \\
\hline 7 & 132 & 108 & 100 & & \\
\hline 8 & 429 & 349 & 315 & 172 & \\
\hline 9 & 1430 & 1144 & 1028 & 980 & \\
\hline 10 & 4862 & 3868 & 3432 & 3240 & 1651 \\
\hline $\mathrm{n}$ & 1 & 2 & 3 & 4 & 5 \\
\hline
\end{tabular}

Table 1: Number of quivers of type $\tilde{A}_{n-1}$ according to the parameter $r$ for $n$ in $\{2,3, \ldots, 10\}$

$r_{1}=s_{1}$ and $r_{2}=s_{2}$ is given by

$$
\begin{aligned}
& 2^{r-2 r_{2}-2}\left(\begin{array}{c}
r \\
r_{2}, r_{2}, r-2 r_{2}
\end{array}\right) \\
&+\sum_{\substack{\left.k|r, k| r_{2} \\
i, j \geq 0 \\
i, j\right) \neq(0,0)}}\left[\frac{\phi(k)}{k} \frac{(-1)^{i+j}}{4(i+j)}\left(\begin{array}{c}
2 i \\
i, 2 i-r / k, r_{2} / k,\left(r-r_{2}\right) / k-i
\end{array}\right)\right. \\
&\left.\quad\left(\begin{array}{c}
2 j \\
j, 2 j-r / k, r_{2} / k,\left(r-r_{2}\right) / k-j
\end{array}\right)\right],
\end{aligned}
$$

where $r=r_{1}+2 r_{2}$.

Proof. According to Theorem 3.5, the expression $\sum_{k \mid r} \frac{\phi(k)}{4 r}\left(\begin{array}{c}2 r / k \\ r / k\end{array}\right)^{2}$ counts realizations of quivers with parameters $r$ and $s=r$. Therefore, it counts non-symmetric quivers with parameters $r$ and $s=r$ twice and symmetric quivers with parameters $r$ and $s=r$ once. By Theorem 3.8, the number of symmetric quivers with parameters $r$ and $s=r$ is given by $\frac{1}{2}\left(\begin{array}{c}2 r \\ r\end{array}\right)$. In total, we get the desired expression. The general case is dealt with similarly.

\subsection{The number of quivers of type $D_{n}$}

With the help of Corollary 3.6 and a little extra work we obtain the number of quivers in the mutation class of Dynkin type $D_{n}$. This result was first determined by Buan 
and Torkildsen in [5].

Corollary 3.10. The number of quivers of type $D_{n}$, for $n \geq 5$, is given by

$$
\tilde{a}(0, n)=\sum_{d \mid n} \frac{\phi(n / d)}{2 n}\left(\begin{array}{c}
2 d \\
d
\end{array}\right) .
$$

The number of quivers of type $D_{4}$ is 6 .

Proof. For $n=4$, the quivers can be explicitly listed, see [5]. We remark that their number does not agree with the general formula. Now, let $\bar{D}_{n}, n \geq 5$, be the family of cyclic arrangements of elements in $B$, with all base arrows oriented clockwise and a total of $n$ vertices. Thus, the elements in $\bar{D}_{n}$ are quivers with a distinguished oriented cycle, which we call the main cycle. Note that the main cycle may be an oriented 2-cycle or even a loop.

We want to show that the quivers of type $D_{n}$ are in bijection with those in $\bar{D}_{n}$. To do so, we use the classification given by Vatne [12], who distinguishes four types $I-I V$. Quivers in $D_{n}$ of type $I V$ coincide with those objects in $\bar{D}_{n}$ whose main cycle consists of at least three arrows. The other three types are as in Figure 3.

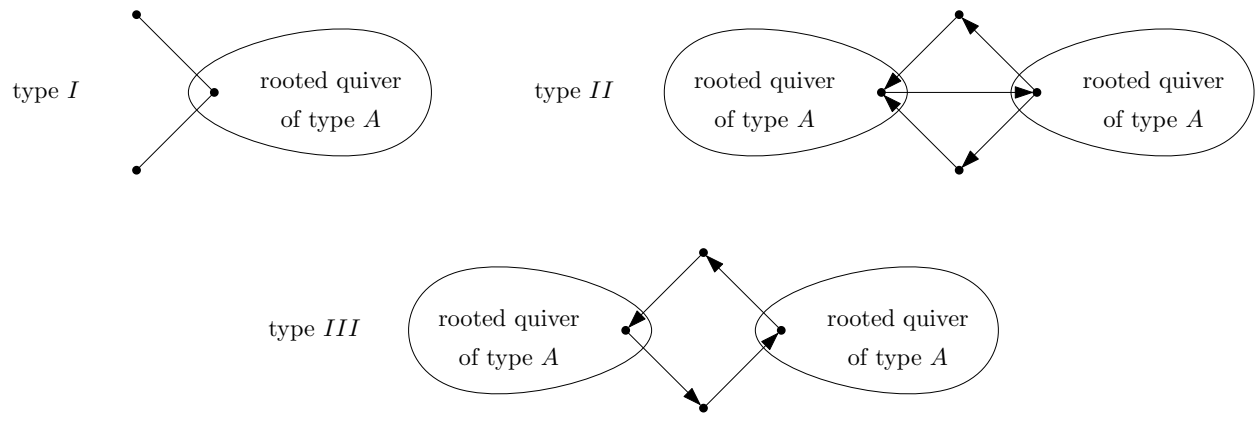

Figure 3: Quivers in $D_{n}$ of type $I-I I I$.

Suppose that the main cycle of $\bar{Q} \in \bar{D}_{n}$ is an oriented 2-cycle. By deleting these two arrows we obtain one of the following:

1. a quiver in $D_{n}$ of type $I$, where precisely one of the two distinguished arrows incident to the root is oriented towards it, or

2. a quiver in $D_{n}$ of type $I I I$, i.e., a quiver having a unique oriented 4-cycle.

The EleCtronic Journal of COMBinatorics 15 (2008), \#R00 
It remains to describe the bijection in the case where the main cycle of $\bar{Q} \in \bar{D}_{n}$ is a loop. In a first step, we delete the vertex of this loop and all arrows incident to it, to obtain a rooted quiver $\bar{Q}^{\bullet}$ of type $A$. For the second and final step, we distinguish two cases:

1. the root of $\bar{Q}^{\bullet}$ is incident to a single arrow $\alpha$. In this case we obtain a quiver $Q$ in $D_{n}$ of type $I$ by adding a second arrow, oriented in the same way as $\alpha$, to the other vertex $\alpha$ is incident to.

2. On the other hand, consider the case that the root of $\bar{Q}^{\bullet}$ is incident to an oriented 3-cycle $\gamma$. Then, we glue a second 3-cycle, oriented in the same way as $\gamma$, along the arrow of $\gamma$ opposite to the root. In this way we create a quiver in $D_{n}$ of type $I I$.

This transformation is invertible:

- a quiver $Q$ in $D_{n}$ of type $I$ has a uniquely determined root, and two distinguished arrows incident to it. If they are oriented in opposite directions, then the main cycle in the preimage of the transformation is an oriented 2-cycle. Otherwise, the preimage is a loop.

- $Q$ is of type $I I$, if and only if it has two oriented 3-cycles sharing an arrow.

- Finally, $Q$ is of type $I I I$, if and only if it has a unique oriented 4-cycle.

To conclude, we compute the number of elements in $\bar{D}_{n}$. This is easy, since we can use the degenerate case of $r=0$ and $s=n$ of Corollary 3.6:

$$
\begin{aligned}
\tilde{a}(0, n) & =\frac{1}{2} \sum_{k \mid n} \frac{\phi(k)}{n}\left(\begin{array}{c}
2 n / k \\
n / k
\end{array}\right) \\
& =\frac{1}{2} \sum_{d \mid n} \frac{\phi(n / d)}{n}\left(\begin{array}{c}
2 d \\
d
\end{array}\right), \quad \text { for } d:=\frac{n}{k} .
\end{aligned}
$$




\section{References}

[1] Ibrahim Assem, Thomas Brüstle, Gabrielle Charbonneau-Jodoin, Pierre-Guy Plamondon, Gentle algebras arising from surface triangulations, Algebra \& Number Theory 4 (2010), no. 2, 201-229, math.RT/0903.3347.

[2] Janine Bastian, Mutation classes of $\tilde{A}_{n}$-quivers and derived equivalence classification of cluster tilted algebras of type $\tilde{A}_{n}$, to appear in Algebra \& Number Theory (2010), 24 pp., math.RT/0901.1515.

[3] François Bergeron, Gilbert Labelle, and Pierre Leroux, Combinatorial species and tree-like structures, Encyclopedia of Mathematics and its Applications, vol. 67, Cambridge University Press, Cambridge, 1998, Translated from the 1994 French original by Margaret Readdy, With a foreword by Gian-Carlo Rota.

[4] Aslak Bakke Buan, Robert Marsh and Idun Reiten, Cluster mutation via quiver representations, Commentarii Mathematici Helvetici 83 (2008), no. 1, 143-177, math.RT/0412077.

[5] Aslak Bakke Buan and Hermund André Torkildsen, The Number of Elements in the Mutation Class of a Quiver of Type $D_{n}$, Electronic Journal of Combinatorics 16 (2009), no. 1, Research Paper 49, 23 pp. (electronic), math.RT/0812.2240.

[6] Aslak Bakke Buan and Dagfinn F. Vatne, Derived equivalence classification for cluster-tilted algebras of type $A_{n}$, Journal of Algebra 319 (2008), no. 7, 27232738, math.RT/0701612.

[7] Philippe Caldero, Frédéric Chapoton, and Ralf Schiffler, Quivers with relations arising from clusters ( $A_{n}$ case), Transactions of the American Mathematical Society 358 (2006), no. 3, 1347-1364, math.RT/0401316.

[8] Philippe Flajolet and Robert Sedgewick, Analytic combinatorics, Cambridge University Press, Cambridge, 2009.

[9] Sergey Fomin, Michael Shapiro, and Dylan Thurston, Cluster algebras and triangulated surfaces. I. Cluster complexes, Acta Mathematica 201 (2008), no. 1, 83-146, math.RA/0608367.

[10] Sergey Fomin and Andrei Zelevinsky, Cluster algebras. I. Foundations, Journal of the American Mathematical Society 15 (2002), no. 2, 497-529 (electronic), math.RT/0104151.

[11] Hermund André Torkildsen, Counting cluster-tilted algebras of type $A_{n}$, International Electronic Journal of Algebra 4 (2008), 149-158, math.RT/0801.3762.

[12] Dagfinn F. Vatne, The mutation class of $D_{n}$ quivers, Comm. Algebra 38 (2010), no. 3, 1137-1146, math.CO/0810.4789. 